\title{
Evaluation of COVID-19 protease and HIV inhibitors interactions
}

\author{
LINH TRAN ${ }^{1,2, *}$ \\ DAO NGOC HIEN TAM ${ }^{3}$ \\ HEBA ELHADAD \\ NGUYEN MINH HIEN ${ }^{5}$ \\ NGUYEN TIEN HUY ${ }^{6}$ \\ ${ }^{1}$ Institute of Fundamental and Applied Sciences \\ Duy Tan University, Ho Chi Minh City 700000 \\ Vietnam \\ ${ }^{2}$ Faculty of Natural Sciences, Duy Tan University \\ Da Nang City, 550000, Vietnam \\ ${ }^{3}$ Asia Shine Trading \& Service Co. Ltd., Ho Chi \\ Minh City, 700000, Vietnam \\ ${ }^{4}$ Department of Parasitology, Medical Research \\ Institute, Alexandria University, Alexandria, Egypt \\ ${ }^{5}$ School of Medicine, Vietnam National University \\ Ho Chi Minh City, Vietnam \\ ${ }^{6}$ Department of Clinical Product Development, \\ Institute of Tropical Medicine, School of Tropical \\ Medicine and Global Health, Nagasaki University \\ Nagasaki 852-8523, Japan \\ Accepted February 27, 2021 \\ Published online April 22, 2021
}

\begin{abstract}
The epidemic of the novel coronavirus disease (COVID-19) that started in 2019 has evoked an urgent demand for finding new potential therapeutic agents. In this study, we performed a molecular docking of anti-HIV drugs to refine HIV protease inhibitors and nucleotide analogues to target COVID-19. The evaluation was based on docking scores calculated by AutoDock Vina and top binding poses were analyzed. Our results suggested that lopinavir, darunavir, atazanavir, remdesivir, and tipranavir have the best binding affinity for the 3-chymotrypsin-like protease of COVID-19. The comparison of the binding sites of three drugs, namely, darunavir, atazanavir and remdesivir, showed an overlap region of the protein pocket. Our study showed a strong affinity between lopinavir, darunavir, atazanavir, tipranavir and COVID-19 protease. However, their efficacy should be confirmed by in vitro studies since there are concerns related to interference with their active sites.
\end{abstract}

Keywords: COVID-19, SARS-CoV-2, docking study, anti-HIV drugs, protease inhibitors

\section{INTRODUCTION}

COVID-19 is an RNA virus belonging to the Coronavirus family. Its sequence was analyzed and published briefly after its emergence (1). The viral genome encodes four non-structural proteins which are essential for the viral life cycle and one structural protein (spike glycoprotein) which is necessary for the infection of host cells. Therefore, the inhibition of these targets might be beneficial for the prevention of viral multiplication and transmission. The COVID-19 sequence was found to be significantly similar to SARS-CoV and MERS-CoV which caused the outbreaks in 2002 and 2012, resp. (2). Therefore, there is an expectation that SARS-CoV or MERS-CoV medicines could be effective in preventing the spread of COVID-19.

\footnotetext{
*Correspondence; e-mail: trantthuylinh10@duytan.edu.vn
} 
Despite a difference between COVID-19 and HIV genomes, successful treatment of COVID-19 with lopinavir/ritonavir; approved anti-HIV drugs, was recently reported $(3,4)$. This combination was also hypothesized to target a non-structural protein named 3-chymotrypsin-like (3CL) protease of SARS and MERS viruses and was associated with improved clinical outcomes in infected patients $(1,5,6)$. Nucleoside reverse transcriptase (NRT), an enzyme that mediates replication of RNA viruses, has also been a major target for many antiviral drugs. Remdesivir is one of the nucleoside reverse transcriptase inhibitors (NRTI) that was used as a potential antiviral drug against a wide array of RNA viruses [including SARS/MERS-CoV, HIV, hepatitis B virus (HBV) or hepatitis C virus (HCV)] with promising results (6). Lopinavir/ritonavir [known as anti-protease inhibitors (PIs)] and remdesivir (known as an NRTI) were screened for COVID-19 treatment, or even for SARS-CoV or MERS-CoV at the early stage of the diseases. To provide more evidence for the possibility of COVID-19 treatment with anti-HIV drugs, this study was conducted to examine the binding affinity of the approved anti-HIV drugs and to evaluate the potential COVID-19 drug candidates using computational approaches.

\section{EXPERIMENTAL}

\section{Protein and ligands preparation}

The 3CL protease structure (PDB ID: 6LU7), the main protease $\left(\mathrm{M}^{\mathrm{pro}}\right)$ which plays an important role in the virus life cycle, was chosen as our target (7). We examined the FDAapproved drugs of HIV PIs including atazanavir (atazanavir sulfate, ATV), darunavir (darunavir ethanolate, DRV), fosamprenavir (fosamprenavir calcium, FOS-APV, FPV), ritonavir (RTV), saquinavir (saquinavir mesylate, SQV), tipranavir (TPV) and lopinavir (8). Regarding the NRTIs, we have examined remdesivir, abacavir (abacavir sulfate, $A B C$ ), emtricitabine (FTC), lamivudine (3TC), tenofovir disoproxil fumarate (tenofovir DF, TDF) and zidovudine (azidothymidine, AZT, ZDV). These drugs were taken from the PubChem database (9) and then converted to three-dimensional structures using OpenBabel software version 2.3.2. (10).

\section{Docking investigation}

All inhibitors were docked into the target protein using Autodock Vina version 1.1.2. (11). To optimize the results, the exhaustiveness of the global search was set as 400 whereas the maximum energy difference was selected. Ten binding modes of docking were generated with random starting positions of ligand, which have fully flexible torsion degrees of freedom. Positions of all atoms of the receptor were kept rigid. The center of grids was placed at the center of the mass of the receptor and the grid dimension was large enough $(80 \times 80 \times 80 \AA)$ to cover the entire 3CL-protease structure.

\section{Docking results analysis}

The docking results were evaluated by binding affinities and the interactions between protein and ligands as well as the favorable binding sites, which were likely to reproduce the experimental data and assess the precision of ligand preparation and docking method. Herein, we used N3 inhibitor (extracted from PDB 6LU7) as a positive control docking. To 
obtain molecular insights into the 3CL protease binding site and the inhibition mechanism, we analyzed the docking results of the top binding poses. A schematic diagram of networks of non-bonded contacts and hydrogen bonds between the ligands and protein binding site was prepared by LigPlot+ version 1.4.5 6. (12).

\section{RESULTS AND DISCUSSION}

The high binding affinity of PIs

The docking scores of all compounds are provided in Table I. Generally, PIs had a stronger binding affinity to 3CL protease than NRTIs. This finding indicates that PIs could
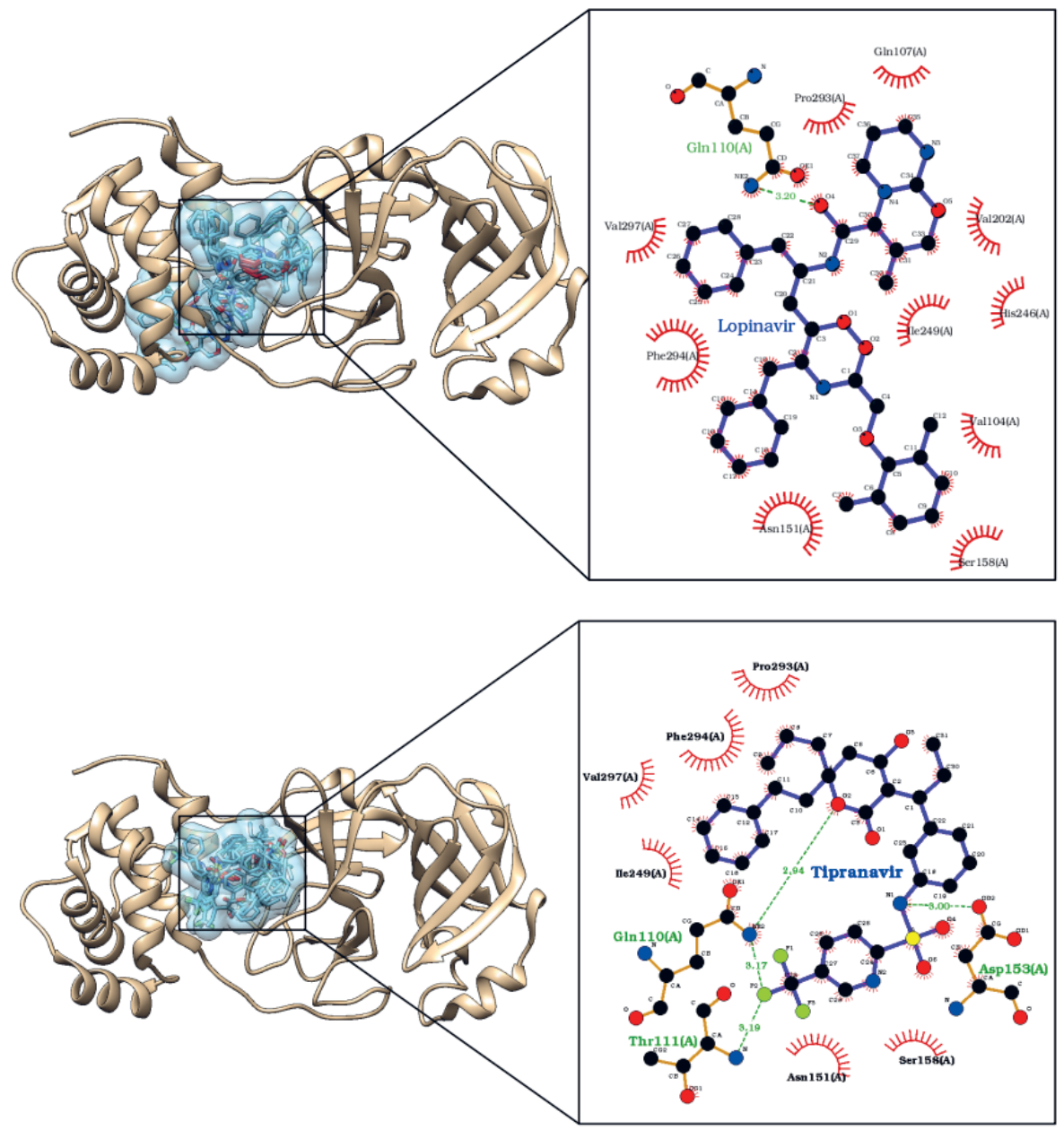

Fig. 1. Binding interactions of lopinavir and tipranavir into 3CL protease. 
Table I. The binding affinity and in vitro results of candidate ligands to 3CL protease

\begin{tabular}{|c|c|c|}
\hline Protease inhibitors (PIs) & $\begin{array}{l}\text { Binding affinity } \\
\left(\mathrm{kcal} \mathrm{mol}^{-1}\right)\end{array}$ & $\begin{array}{c}I C_{50} \\
\left.(\mu \mathrm{mol} \mathrm{L})^{-1}\right)\end{array}$ \\
\hline Lopinavir & -9.2 & NA \\
\hline Darunavir (darunavir ethanolate, DRV) & -9.1 & $36.1 \pm 1.2$ \\
\hline Atazanavir (atazanavir sulfate, ATV) & -8.9 & $60.7 \pm 2.5$ \\
\hline Tipranavir (TPV) & -8.4 & $\begin{array}{c}\text { No inhibition } \\
\text { (up to } 200 \mu \mathrm{mol} \mathrm{L}^{-1} \text { ) }\end{array}$ \\
\hline Ritonavir (RTV) & -8.2 & $13.7 \pm 1.1$ \\
\hline Fosamprenavir (fosamprenavir calcium, FOS-APV, FPV) & -8.2 & NA \\
\hline Saquinavir (saquinavir mesylate, SQV) & -7.9 & $31.4 \pm 1.2$ \\
\hline \multicolumn{3}{|l|}{ Control docking } \\
\hline N3 inhibitor & -7.4 & NA \\
\hline \multicolumn{3}{|l|}{ Nucleoside reverse transcriptase inhibitors (NRTIs) } \\
\hline Remdesivir & -8.8 & NA \\
\hline Abacavir (abacavir sulfate, $\mathrm{ABC}$ ) & -7.6 & NA \\
\hline Tenofovir Disoproxil Fumarate (tenofovir DF, TDF) & -6.6 & NA \\
\hline Zidovudine (azidothymidine, AZT, ZDV) & -6.5 & NA \\
\hline Emtricitabine (FTC) & -6 & NA \\
\hline Lamivudine (3TC) & -5.8 & NA \\
\hline
\end{tabular}

NA - no activity

exhibit a stronger inhibitory activity than NRTIs. The top five binding scores were of lopinavir, darunavir, atazanavir, remdesivir, and tipranavir. In detail, the binding affinity of lopinavir was the highest $\left(-9.2 \mathrm{kcal} \mathrm{mol}^{-1}\right)$, followed by darunavir $\left(-9.1 \mathrm{kcal} \mathrm{mol}^{-1}\right)$, atazana$\operatorname{vir}\left(-8.9 \mathrm{kcal} \mathrm{mol}^{-1}\right)$, remdesivir $\left(-8.8 \mathrm{kcal} \mathrm{mol}^{-1}\right)$ and tipranavir $\left(-8.4 \mathrm{kcal} \mathrm{mol}^{-1}\right)$.

\section{Molecular interactions of COVID-19 protease and top binding inhibitors}

The binding sites were highly sensitive to the targets. The binding sites of the top five inhibitors are shown in Fig. 1. It was observed that lopinavir and tipranavir had the same binding site, meanwhile, darunavir, atazanavir, remdesivir preferentially exposed another binding site similar to the docking control (N3 inhibitor). In the case of lopinavir interaction, the highest binding position is created by a hydrogen bond at Gln110 and several non-bonded contacts are Val104, Gln107, Asn151, Ser158, Val202, His246, Ile249, Phe294, Val297 and Pro293. Similar to lopinavir, the binding position of tipranavir was found by three hydrogen bonds including Gln110, Thr111 and Asp153, with the non-bonded contact network of Asn151, Ser158, Ile249, Pro293, Phe294, and Val297. For darunavir, atazanavir and remdesivir, we found an overlapped binding hotspot among the active site of 3CL protease, presented in Fig. 2. The common significant binding hotspot residues for daruna- 

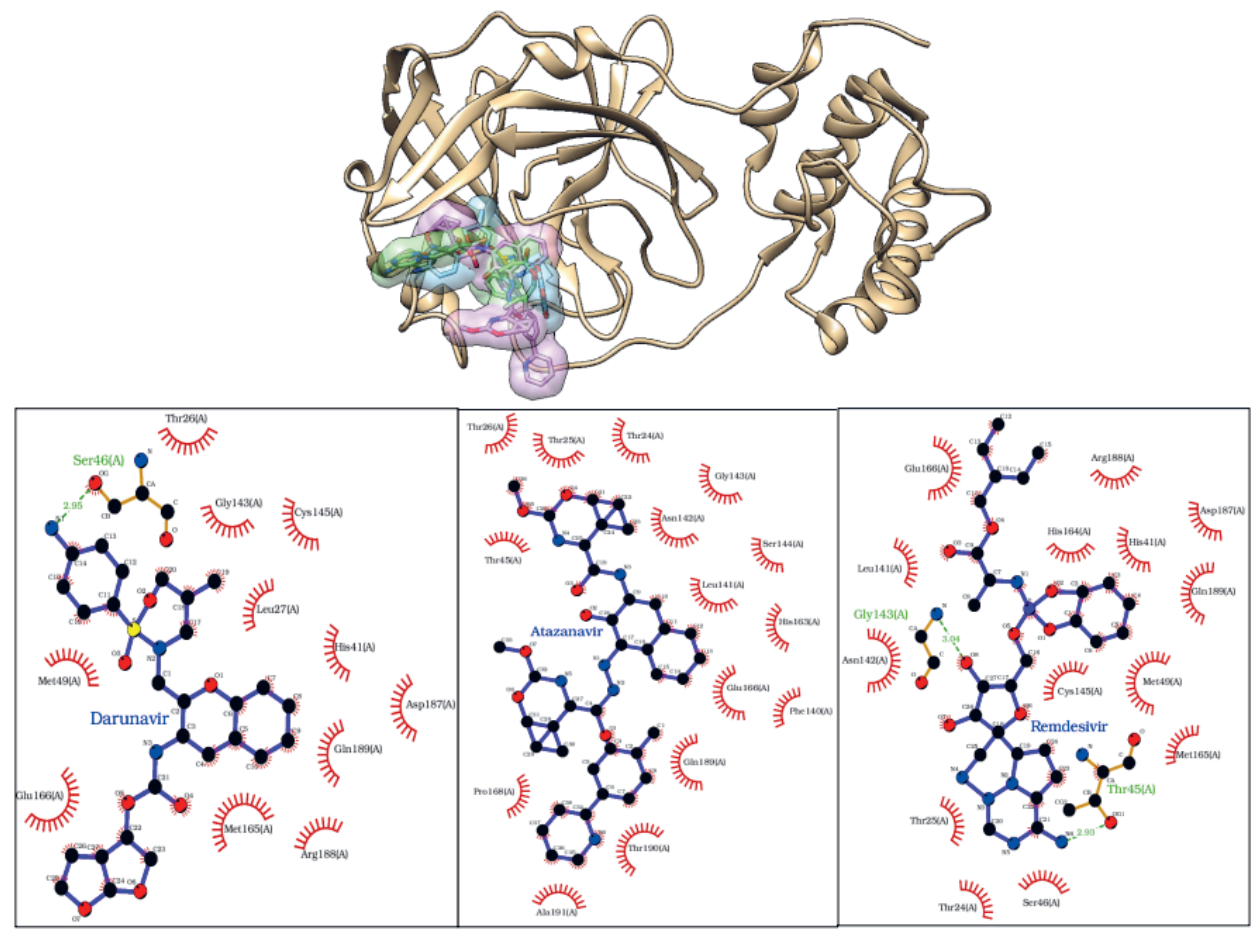

Fig. 2. The overlap of binding positions of darunavir, atazanavir, and remdesivir into 3CL protease at the active site.

vir, atazanavir, and remdesivir were as follows: His41, Met49, Leu141, Asn142, His164, Met165, Glu166, and Gln189. Atazanavir had no hydrogen bond, meanwhile, darunavir obtained a hydrogen bond with Ser46 and remdesivir created two hydrogen bonds with Thr45 and Gly143. Because the 3CL protease does not have a well-defined binding site, the top five inhibitors were located at different positions, as they preferred to interact closely to several hydrophobic residues.

\section{DISCUSSION}

Our results showed that some anti-HIV medicines had a strong affinity to 3CL protease of COVID-19 in silico model, including lopinavir, darunavir, atazanavir, remdesivir and tipranavir. These drugs show their effect by inhibition of HIV protease via blocking its active site, thus inhibiting the viral multiplication (8). Amongst them, the combination of lopinavir/ritonavir is being applied for COVID-19 treatment in China and showed positive recovery of patients, although the structure of HIV protease and COVID-19 are not exactly the same (13). The role of ritonavir is to inhibit cytochrome P450 (CYP) 3A4 resulting in slow elimination of lopinavir, which is strongly metabolized via CYP3A4, from the body. Therefore, lopinavir plays the main role in this combination for inhibition of viral matura- 
tion. In our study, the strong affinity between lopinavir and 3CL protease might elucidate its effect; this finding was in line with the previous study (14). The chemical structure of lopinavir appeared to be fixed inside the viral protein that could cause strong binding as well as the long-term interaction between them. However, there was a concern relating to its ability to reduce viral replication when tested on MERS-CoV. This combination appeared to improve the symptoms rather than kill the virus. Its pharmacokinetic characteristics revealed binding of the majority of lopinavir to plasma proteins, which means that only a minor free unbound level of the drug is available to exert its effect. Fortunately, the in vitro maximal effective concentration $\left(E C_{50}\right)$ against MERS-CoV of this treatment was within the average level of the free drug in the body. Until now, the current reports indicated that the symptoms of COVID-19 patients have improved but there is no report of viral elimination relating to this treatment $(15,16)$. A recent in vitro study reported that the $I C_{50}$ value of lopinavir in inhibiting protease was about $500 \mu \mathrm{mol} \mathrm{L}^{-1}$ (17). Taking all together, we recommend screening of other NRTIs with good bioavailability and high levels of the free active form in the body.

Our findings were in line with another study that used another in silico model about the potency of atazanavir (18). This study showed that atazanavir might be the best candidate, as it had the highest inhibitory potency amongst the tested structures. The inhibitory potency values in this prediction model could not exactly reflect the potency of a drug in clinical practice, as the efficacy of lopinavir/ritonavir was predicted to be weak. However, due to the consistency in prediction between the two studies, atazanavir could become a potential candidate in screening for new COVID-19 medicines. This drug also has satisfactory pharmacokinetic characteristics, with modest bioavailability and a high percent of unchanged form in the body (19). On the other hand, a previous screening reported that atazanavir was ineffective for the treatment of MERS-CoV; therefore, in vitro or in vivo studies should be conducted to examine its efficacy before applying it into clinical practices (16).

Darunavir had a stronger affinity to 3CL protease than atazanavir, but its pharmacokinetic characteristics are not very prominent, as it shows fast absorption with fast distribution and elimination as well (20). The percentage of its free form level is also quite small, similar to lopinavir. Therefore, we cannot ensure whether it could reach the feasible concentration required to exert its effect. It is worthy to mention that a previous in vitro study conducted by Mahdi et al. (21) reported that lopinavir, ritonavir, darunavir, saquinavir and atazanavir were able to inhibit SARS-CoV-2 main viral protease in the cell culture, albeit in concentrations much higher than their achievable plasma levels. They stated that inhibition by lopinavir was attributed to its cytotoxicity, while ritonavir was the most effective of the panel, with $I C_{50}$ of $13.7 \mu \mathrm{mol} \mathrm{L}-1$. On the other hand, atazanavir was the only PI to inhibit the viral protease both in cell culture and in in vitro enzymatic assay.

Despite our findings could suggest potential candidates that had a strong binding affinity to $3 \mathrm{CL}$ protease, being potential agents against SARS-CoV-2, we recommend all compounds (even those that had a weaker affinity to 3CL protease) to be tested, at least in vitro, to affirm their activity (22-24). A virtual screening study has identified 14 known antiviral drugs with possible inhibitory effects on the main protease of SARS-CoV-2 (24). Moreover, besides 3CL protease, other proteinases could become targets for screening agents against SAR-CoV-2. These targets are papain-like protease, helicase, RNA-dependent RNA polymerase, and spike glycoprotein (1). Therefore, we recommend further stu- 
dies to determine the interaction of other candidates and the mentioned targets in order to find out potential drugs.

For instance, remdesivir has a lower affinity to 3CL proteinase compared to lopinavir, darunavir or atazanavir, but it has a good effect in clinical practice because it is active through its inhibition of reverse transcriptase, rather than viral proteinase such as $3 \mathrm{CL}$ proteinase.

\section{CONCLUSIONS}

In our work, five anti-HIV inhibitors, namely, lopinavir, darunavir, atazanavir, remdesivir, and tipranavir were identified to have potential inhibitory activities against COVID-19 protease. A set of possible top hits binding interactions between 3CL protease and anti-HIV drugs could substantially contribute to the rational drug development against COVID-19.

\section{REFERENCES}

1. F. Wu, S. Zhao, B. Yu, Y.-M. Chen, W. Wang, Z.-G. Song, Y. Hu, Z.-W. Tao, J.-H. Tian, Y.-Y. Pei, M.-L. Yuan, Y.-L. Zhang, F.-H. Dai, Y. Liu, Q.-M. Wang, J.-J. Zheng, L. Xu, E. C. Holmes and Y.-Z. Zhang, A new coronavirus associated with human respiratory disease in China, Nature 579 (2020) 265-269; https://doi.org/10.1038/s41586-020-2008-3

2. J. Xu, S. Zhao, T. Teng, A. E. Abdalla, W. Zhu, L. Xie, Y. Wang and X. Guo, Systematic comparison of two animal-to-human transmitted human coronaviruses: SARS-CoV-2 and SARS-CoV, Viruses 12 (2020) Article ID 244; https://doi.org/10.3390/v12020244

3. Z. Wang, X. Chen, Y. Lu, F. Chen and W. Zhang, Clinical characteristics and therapeutic procedure for four cases with 2019 novel coronavirus pneumonia receiving combined Chinese and Western medicine treatment, Biosci. Trends 14 (2020) 64-68; https://doi.org/10.5582/bst.2020.01030

4. J. Lim, S. Jeon, H. Y. Shin, M. J. Kim, Y. M. Seong, W. J. Lee, K. W. Choe, Y. M. Kang, B. Lee and S. J. Park, Case of the index patient who caused tertiary transmission of COVID-19 infection in Korea: the application of lopinavir/ritonavir for the treatment of COVID-19 infected pneumonia monitored by quantitative RT-PCR, J. Korean Med. Sci. 35 (2020) e79; https://doi.org/10.3346/jkms.2020.35.e79

5. C. M. Chu, V. C. Cheng, I. F. Hung, M. M. Wong, K. H. Chan, K. S. Chan, R. Y. Kao, L. L. Poon, C. L. Wong, Y. Guan, J. S. Peiris and K. Y. Yuen, Role of lopinavir/ritonavir in the treatment of SARS: initial virological and clinical findings, Thorax 59 (2004) 252-256; https://doi.org/10.1136/thorax.2003.012658

6. S. J. Hurwitz and R. F. Schinazi, Practical considerations for developing nucleoside reverse transcriptase inhibitors, Drug Discov. Today Technol. 9 (2012) e183-e193; https://doi.org/10.1016/j. ddtec.2012.09.003

7. N. Atatreh, S. Hasan, B. R. Ali and M. A. Ghattas, Computer-aided approaches reveal trihydroxychroman and pyrazolone derivatives as potential inhibitors of SARS-CoV-2 virus main protease, Acta Pharm. 71 (2021) 325-333; https://doi.org/10.2478/acph-2021-0040

8. Z. Lv, Y. Chu and Y. Wang, HIV protease inhibitors: a review of molecular selectivity and toxicity, HIVIAIDS (Auckland) 7 (2015) 95-104; https://doi.org/10.2147/HIV.S79956

9. Y. Wang, J. Xiao, T. O. Suzek, J. Zhang, J. Wang, Z. Zhou, L. Han, K. Karapetyan, S. Dracheva, B. A. Shoemaker, E. Bolton, A. Gindulyte and S. H. Bryant, PubChem's BioAssay Database, Nucleic Acids Res. 40 (2012) D400-D412; https://doi.org/10.1093/nar/gkr1132 
10. N. M. O’Boyle, M. Banck, C. A. James, C. Morley, T. Vandermeersch and G. R. Hutchison, Open Babel: An open chemical toolbox, J. Cheminform. 3 (2011) Article ID 33; https://doi.org/10.1186/17582946-3-33

11. O. Trott and A. J. Olson, AutoDock Vina: improving the speed and accuracy of docking with a new scoring function, efficient optimization, and multithreading, J. Comput. Chem. 31 (2010) 455-461; https://doi.org/10.1002/jcc.21334

12. R. A. Laskowski and M. B. Swindells, LigPlot+: multiple ligand-protein interaction diagrams for drug discovery, J. Chem. Inf. Model. 51 (2011) 2778-2786; https://doi.org/10.1021/ci200227u

13. M. Wang, R. Cao, L. Zhang, X. Yang, J. Liu, M. Xu, Z. Shi, Z. Hu, W. Zhong and G. Xiao, Remdesivir and chloroquine effectively inhibit the recently emerged novel coronavirus (2019-nCoV) in vitro, Cell Res. 30 (2020) 269-271; https://doi.org/10.1038/s41422-020-0282-0

14. S. Daoud, S. J. Alabed, L. A. Dahabiyeh, Identification of potential COVID-19 main protease inhibitors using structure-based pharmacophore approach, molecular docking and repurposing studies, Acta Pharm. 71 (2021) 163-174; https://doi.org/10.2478/acph-2021-0016

15. T. P. Sheahan, A. C. Sims, S. R. Leist, A. Schäfer, J. Won, A. J. Brown, S. A. Montgomery, A. Hogg, D. Babusis, M. O. Clarke, J. E. Spahn, L. Bauer, S. Sellers, D. Porter, J. Y. Feng, T. Cihlar, R. Jordan, M. R. Denison and R.S. Baric, Comparative therapeutic efficacy of remdesivir and combination lopinavir, ritonavir, and interferon beta against MERS-CoV, Nat. Commun. 11 (2020) Article ID 222; https://doi. org/10.1038/s41467-019-13940-6

16. A. H. de Wilde, D. Jochmans, C. C. Posthuma, J. C. Zevenhoven-Dobbe, S. van Nieuwkoop, T. M. Bestebroer, B. G. van den Hoogen, J. Neyts and E. J. Snijder, Screening of an FDA-approved compound library identifies four small-molecule inhibitors of Middle East respiratory syndrome coronavirus replication in cell culture, Antimicrob. Agents Chemother. 58 (2014) 4875-4884; https://doi. org/10.1128/AAC.03011-14

17. E. C. Vatansever, K. S. Yang, K. Kratch, A. Drelich, C.-C. Cho, D. M. Mellot, S. Xu, C.-T. K. Tseng and W. R. Liu, Targeting the SARS-CoV-2 main protease to repurpose drugs for COVID-19, bioRxiv preprint [Internet], posted May 23, 2020; https://doi.org/10.1101/2020.05.23.112235

18. B. R. Beck, B. Shin, Y. Choi, S. Park and K. Kang, Predicting commercially available antiviral drugs that may act on the novel coronavirus (SARS-CoV-2) through a drug-target interaction deep learning model, Comput. Struct. Biotechnol. J. 18 (2020) 784-790; https://doi.org/10.1016/j.csbj.2020.03.025

19. M. A. M. Subbaiah, S. Mandlekar, S. Desikan, T. Ramar, L. Subramani, M. Annadurai, S. D. Desai, S. Sinha, S. M. Jenkins and M. R. Krystal, Design, synthesis, and pharmacokinetic evaluation of phosphate and amino acid ester prodrugs for improving the oral bioavailability of the HIV-1 protease inhibitor atazanavir, J. Med. Chem. 62 (2019) 3553-3574; https://doi.org/10.1021/acs. jmedchem.9b00002

20. M. Rittweger and K. Arastéh, Clinical pharmacokinetics of darunavir, Clin. Pharmacokin. 46 (2007) 739-756; https://doi.org/10.2165/00003088-200746090-00002

21. M. Mahdi, J. A. Mótyán, Z. I. Szojka, M. Golda, M. Miczi and J. Tőzsér, Analysis of the efficacy of HIV protease inhibitors against SARS-CoV-2's main protease, Virol. J. 17 (2020) Article ID 190; https:// doi.org/10.1186/s12985-020-01457-0

22. S. Jo, S. Kim and D. H. Shin, Inhibition of SARS-CoV 3CL protease by flavonoids, J. Enzyme Inhib. Med. Chem. 35 (2020) 145-151; https://doi.org/10.1080/14756366.2019.1690480

23. L. Zhang, D. Lin, Y. Kusov, Y. Nian, Q. Ma, J. Wang, A. von Brunn, P. Leyssen, K. Lanko, J. Neyts, A. de Wilde, E. J. Snijder and H. Liu, $\alpha$-ketoamides as broad-spectrum inhibitors of coronavirus and enterovirus replication: structure-based design, synthesis, and activity assessment, J. Med. Chem. 63 (2020) 4562-4578; https://doi.org/10.1021/acs.jmedchem.9b01828

24. M. M. Ghahremanpour, J. Tirado-Rives, M. Deshmukh, J. A. Ippolito, C.-H. Zhang, I. Cabeza de Vaca, M.-E. Liosi, K. S. Anderson and W. L. Jorgensen, Identification of 14 known drugs as inhibitors of the main protease of SARS-CoV-2, ACS Med. Chem. Lett. 11 (2020) 2526-2533; https://doi.org/ 10.1021/acsmedchemlett.0c00521 